\title{
MODELAGEM DA CIRCULAÇÃO ATMOSFÉRICA NA REGIÃO DA USINA TERMOELÉTRICA DE CANDIOTA
}

Rita de Cássia Marques Alves e Maria Assunção Faus da Silva Dias

Departamento de Ciências Atmosféricas, Universidade de São Paulo

Rua do Matão 1226, São Paulo, SP, Brasil

Osvaldo Luiz Leal de Moraes

Departamento de Física - CCNE

UFSM - Santa Maria, RS, Brasil

\section{SUMMARY}

In this paper it is used the Regional Atmospheric Modeling System (RAMS) in order to simulate the atmospheric circulation around the Candiota Thermoelectric Power Plant. Two numerical experiments are showed. The first one describes the sensitivity of the model to the local topography. In the second one the model is initialized with data from CPTEC/INPE and the results are compared with data obtained in fields campaigns. This comparison indicates that the RAMS outputs are an useful tools to be used in atmospheric dispersion models to describe the pollutant transport emitted by the Candiota source.

\section{INTRODUÇÃO}

Rev. Ciência e Natura - Dispersion Process: 93 - 2000. 
A poluição atmosférica representa hoje uma desagradável realidade para um número grande de comunidades urbanas e industriais. Os processos de transporte e difusão turbulenta na atmosfera são assuntos de considerável atividade de pesquisa nas áreas de Meteorologia e de Dinâmica de Fluidos. Apesar do grande avanço ocorrido nas últimas duas décadas a dinâmica dos mecanismos de circulação local, transporte médio e difusão turbulenta de poluentes, na Camada Limite Planetária (CLP), está longe de ser completamente compreendida. Os diferentes movimentos atmosféricos são normalmente turbulentos, excitam infinitos graus de liberdade no meio e correlacionam-se de uma maneira completamente não-linear. Os efeitos topográficos e as sensibilidades as condições iniciais nos mecanismos de forçantes podem levar a formação de diferentes tipos de estrutura de fluxos, vinculando assim, no sentido estatístico, as aparições dos fenômenos meteorológicos locais aos tipos de relevos e propriedades físicas do solo.

Muitos programas de pesquisa, envolvendo observações, são realizados em diferentes sítios para determinar a correlação entre difusão atmosférica e variáveis meteorológicas. As relações entre parâmetros meteorológicos e o comportamento difusivo do ar permitem o desenvolvimento de esquemas e modelos matemáticos empregados na predição da dispersão atmosférica.

Neste trabalho o Regional Atmospheric Modeling System (RAMS) é utizido para descrever a circulação atmosférica no sul do estado do Rio Grande do Sul, particularmente na região onde localiza-se a Usina Termoelétrica de Candiota. O objetivo é entender a importância dos processos advectivos na poluição atmosférica causada por aquela fonte. Para tanto dois experimentos numéricos são descritos. O primeiro busca-se entender o papel desempenhado pelas caractéricas locais no terreno no campo de vento. O segundo experimento, simula a circulação para um dia específico e os resultados são comparados com dados coletados em situ durante o Projeto Candiota (Moraes et al 1996, Moraes 2000). 


\section{O PROJETO CANDIOTA}

O objetivo do projeto Candiota foi quantificar o impacto ambiental causado pela Usina Termoelétrica Presidente Médice (UTPM) no sul do Brasil e Uruguai. Para tanto campanhas experimentais, de coleta de dados meteorológicos e de concentração de contaminantes, foram realizadas na região. Os dados obtidos nestas campanhas, realizadas entre Janeiro de 1994 e Agosto de 1999, destinaram-se, em uma primeira fase, a possibilitar a descrição da Camada Limite Planetária local e a circulação atmosférica regional. $\mathrm{Na}$ etapa seguinte, tais descrições possibilitaram a modelagem do transporte dos poluentes, com diferentes modelos, bem como a comparação das predições destes modelos com as concentrações observadas.

A UTPM está localizada em $31.3 \mathrm{~S}-53.5 \mathrm{~W}$ e sua chaminé possui uma altura de 150 metros. O diâmetro da fonte é de 5 metros e os gases são emitidos a uma temperatura de $430 \mathrm{~K}$ e velocidade de $20 \mathrm{~m} / \mathrm{s}$. Com operação máxima (aproximadamente $400 \mathrm{MW}$ ) a taxa de emissão de dióxido de enxofre chega a 2200 gramas por segundo. Importante enfatizar que esta é a única fonte de $\mathrm{SO}_{2}$ na região.

Nas seis campanhas experimentais, pesquisadores de diversas instituições do país estiveram envolvidos (Universidade Federal de Santa Maria, Universidade de São Paulo, Instituto Nacional de Pesquisas Espaciais, Instituto de Pesquisas Meteorológicas de Bauru e Universidade de Rio Grande). Nestas campanhas, medidas superficiais foram feitas em uma torre micrometeorológica equipada com sensores de resposta rápida e resposta lenta e medidas em altitude foram obtidas com radiosondagens, balão cativo e balão piloto. A descrição completa destes experimentos está descrita em Moraes et al (1996). 


\section{O REGIONAL ATMOSPHERIC MODELING SYSTEM (RAMS)}

Para simular a circulação dos movimentos atmosféricos na região de Candiota será utilizado o modelo RAMS (Regional Atmospheric Modeling System), desenvolvido pela Universidade do Estado do Colorado. As principais características deste modelo foram descritas em detalhes por diversos autores a saber: Cotton e Tripoli (1978), Cotton (1980), Tripoli e Cotton (1980), Tripoli e Cotton (1982), Tremback et al. (1985), Tremback et al. (1987), Tremback (1990) e Pielke et al. (1992).

As equações usadas no modelo são aquelas para uma atmosfera não hidrostática e com várias opções de parametrização dos processos associados à difusão turbulenta atmosférica, radiação solar, processos úmidos (que incluem a formação e interação de nuvens e precipitação de hidrometeoros na fase líquida e gelo, convecção de cumulus), troca de calor sensível e latente entre a superfície e atmosfera (incluindo o papel da vegetação) e transporte de calor o solo. O RAMS é um modelo elaborado para ser utilizado em mesoescala mas também pode ser empregado em outras escalas, fazendo-se os ajustes correspondentes a escala de interesse.

No estudo do escoamento com topografia irregular usa-se "sigma" como coordenada vertical. A especificação desta sistema de coordenadas no RAMS segue as considerações de Clark (1977).

A diferenciação numérica no tempo das equações do modelo é efetuada através de um esquema híbrido, que consiste de diferenciação avançada para as variáveis termodinâmicas e de diferenciação do tipo "leapfrog" para as componentes de velocidade e para a pressão. A seguir descreve-se as principais parametrizações utilizadas neste trabalho.

\section{PARAMETRIZAÇÃO DA RADIAÇÃO}

96 Rev. Ciência e Natura - Dispersion Process: 93 - 2000. 
Nas simulações realizadas neste estudo foi utilizado o esquema de parametrização de Chen e Cotton (1983). Para onda curta, este esquema inclui os efeitos do espalhamento Rayleigh, a absorção pelo vapor d'água e a inclinação do terreno; enquanto que o de onda longa, inclui a emissividade do vapor d'água e do dióxido de carbono. Além disso, são considerados os efeitos radiativos da água líquida e do gelo.

\section{PARAMETRIZAÇÃO DA TURBULÊNCIA}

A parametrização da turbulência usada no modelo RAMS tem como base a deformação anisotrópica (Stull, 1988). O termo de fluxo turbulento $\overline{\mathrm{A} " \mathrm{u} " \mathrm{j}}$ é parametrizado segundo a teoria $\mathrm{K}$ de deformação (Smagorinski, 1963) através de um fechamento de viscosidade turbulenta. A difusão vertical é obtida através de uma analogia unidimensional com o esquema de Smagorinski (1963). Na horizontal, a deformação é calculada através de um fechamento de primeira ordem, que utiliza equações prognósticas para os campos médios em todas as variáveis, enquanto os termos de segunda ordem são parametrizados. Os coeficientes horizontais de difusão são calculados pelo produto entre a taxa de deformação horizontal, obtido pelo gradiente horizontal de velocidade, e a escala de comprimento ao quadrado, dada por $\mathrm{I}=\Delta \mathrm{x} . \mathrm{csx}$. Da mesma forma, a difusão vertical é obtida analoga ao esquema de Smagorinsky em uma dimensão, no qual a deformação vertical é avaliada através dos gradientes verticais do vento horizontal e da escala de comprimento, dada por I $=\Delta z$.csz.

\section{MODELO DE SOLO E VEGETAÇÃO}


O modelo de solo e vegetação utilizado pelo modelo RAMS foi previamente desenvolvido por McCumber e Pielke (1981) e implementado posteriormente por Tremback e Kessler (1985).

A temperatura da superfície é calculada através do balanço de energia aplicado à interface superfície-atmosfera de acordo com a seguinte equação:

$$
R_{n}+\rho \mathrm{L} u_{*} q_{*}+\rho c_{p} u_{*} \Theta_{*}-G=0
$$

onde o primeiro termo é o fluxo de radiação líquida na superfície, o segundo é o fluxo turbulento de calor latente, o terceiro de calor sensível e o quarto é o fluxo de calor no solo. As quantidades turbulentas $u^{*}, q^{*}$ e $\theta^{*}$ são expressas como (2), e representam, respectivamente o momento, o calor e a umidade turbulenta na superfície:

$$
\begin{aligned}
u_{*} & =\frac{k_{0}\left(u^{2}+v^{2}\right)^{1 / 2}}{\left[\ln \left(z / z_{0}\right)-I_{1}\right]} \\
\theta^{*} & =\frac{k_{0}\left(\theta-\theta\left(z_{0}\right)\right.}{\left\{0,74\left[\ln \left(z / z_{0}\right)-I_{2}\right]\right\}} \\
q^{*} & =\frac{k_{0}\left(q-q\left(z_{0}\right)\right.}{\left\{0,74\left[\ln \left(z / z_{0}\right)-I_{3}\right]\right\}}
\end{aligned}
$$

onde $\mathrm{k}_{0}$ é a constante de von Karman e $\mathrm{z}_{0}$ é a altura de rugosidade turbulenta. As quantidades $I_{1}, I_{2}$ e $I_{3}$ representam ajustes baseados na estabilidade.

Abaixo da interface superfície-atmosfera, o transporte é feito pela difusão vertical de calor dada por:

$$
C \frac{\partial T_{s}}{\partial t}=\frac{\partial H_{s}}{\partial t}
$$

onde $\mathrm{H}_{\mathrm{s}}$ representa o fluxo de calor no solo e $\mathrm{C}$ é a capacidade volumétrica.

A representação da vegetação usada no RAMS é basicamente a parametrização de uma camada volumétrica, descrita por Avissar e Pielke 98 Rev. Ciência e Natura - Dispersion Process: 93 - 2000. 
(1989). Assumindo que a capacidade calorífica da vegetação é pequena quando comparado aos fluxos de energia, o balanço de energia nesta camada é dado por:

$$
R_{N v}+E_{v}+H_{v}=0
$$

onde $R_{N_{v}}$ é a radiação líquida do dossel, $E_{v}$ e $H_{v}$ são os fluxo de calor latente e sensível entre a vegetação e o ar ambiente.

\section{RESULTADOS}

Nesta seção são apresentados resultados de dois diferentes experimentos numéricos. O primeiro objetiva verificar a importância dos forçantes topográficos para a circulação atmosférica na região. Em outras palavras: busca-se conhecer como os perfis de vento, temperatura e umidade são influenciados pela orografia local. O segundo experimento é uma validação do modelo, isto é: o RAMS é inicializado com dados fornecidos pelo CPTEC/INPE e os resultados são comparados com dados coletados in situ.

Nas duas simulações o modelo está centrado na latitude -31.3 e longitude -53.5 , localização da cidade de Candiota-RS. A grade tem 50 pontos nas direções horizontais, com espaçamento de $25 \mathrm{Km}$. Na vertical são 30 pontos e espaçamento $\Delta Z=20$ metros, com fator 1.2 na grade telescópica, até atingir 1000 metros de espessura, permanecendo constante até o topo do modelo. O tempo de simulação é de 24 horas, com saída a cada uma hora. A parametrização de solo-vegetação considerada é tipo grama e a parametrização de radiação é a proposta por Chen e Cotton (1983). A parametrização de turbulência é a de Smagorinsky (1963).

As figuras abaixo mostram a topografia na região e um corte longitudinal, isto é, fixando-se a latitude em $31.3 \mathrm{~S}$ e variando-se a longitude. $\mathrm{Na}$ figura 2, em 50.6W está o limite entre o oceano e o continente. A lagoa 
dos Patos está entre 51.6 e 51.8 W. Em 53.5 W localiza-se Candiota e em 58.2 W está o rio Uruguai.

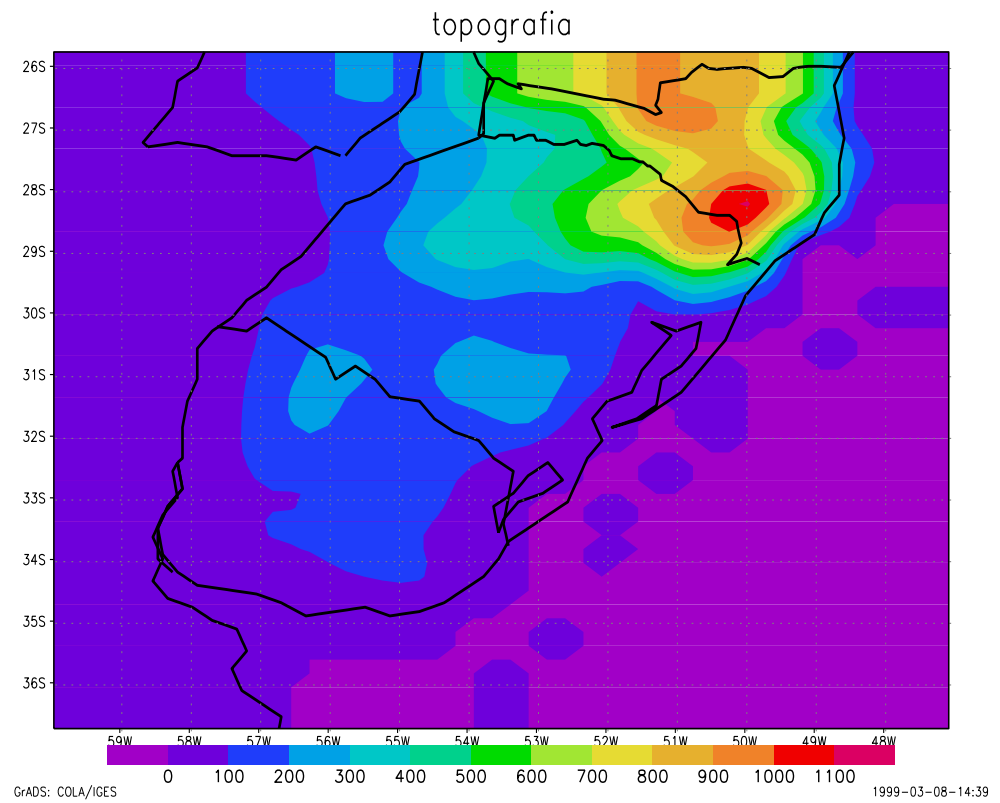

Figura 1 - Topografia na área de abrangência da simulação. 


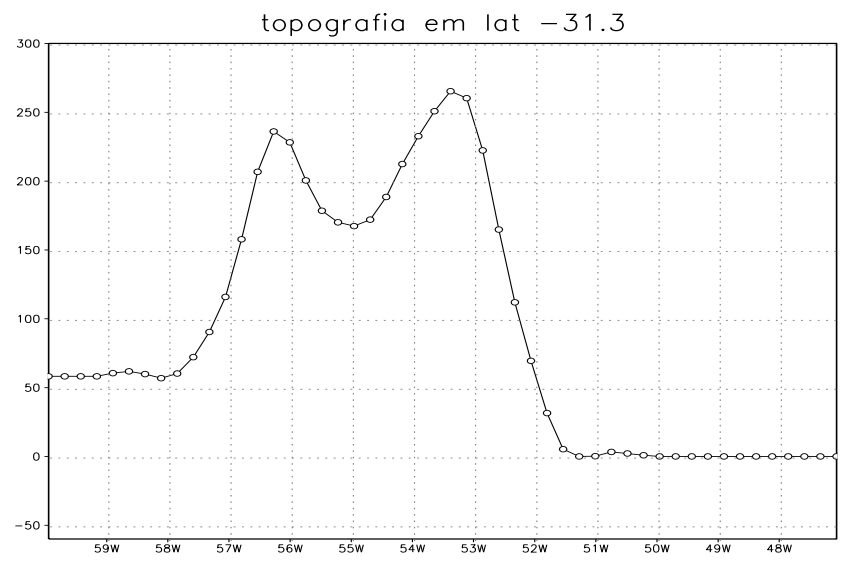

GrADS: COLA/IGES

Figura 2: Corte longitudinal da figura 1, na latitude -31.3. O Oceano Atlântico está a direita de $50 \mathrm{~W}$. Altitude em Candiota (53.5W) é de 250 metros. Em 58W localiza-se o rio Uruguai.

\section{CIRCULAÇÕES INDUZIDAS POR FORÇANTES TOPOGRÁFICOS}

O modelo é inicializado com perfil de temperatura, umidade e pressão obtidos de uma radiosondagem do dia 09/11/1995 as seis horas local. A velocidade e direção do vento, em todo o domínio, foram de $0,5 \mathrm{~m} / \mathrm{s}$ e 0 graus respectivamente. Essa inicialização foi utilizada com o objetivo de forçar a ocorrência da brisa, conforme sugerido por Avissar and Chen (1993).

As figuras a seguir ilustram o campo de vento em superfície e em 1100 metros as 12 horas local. 


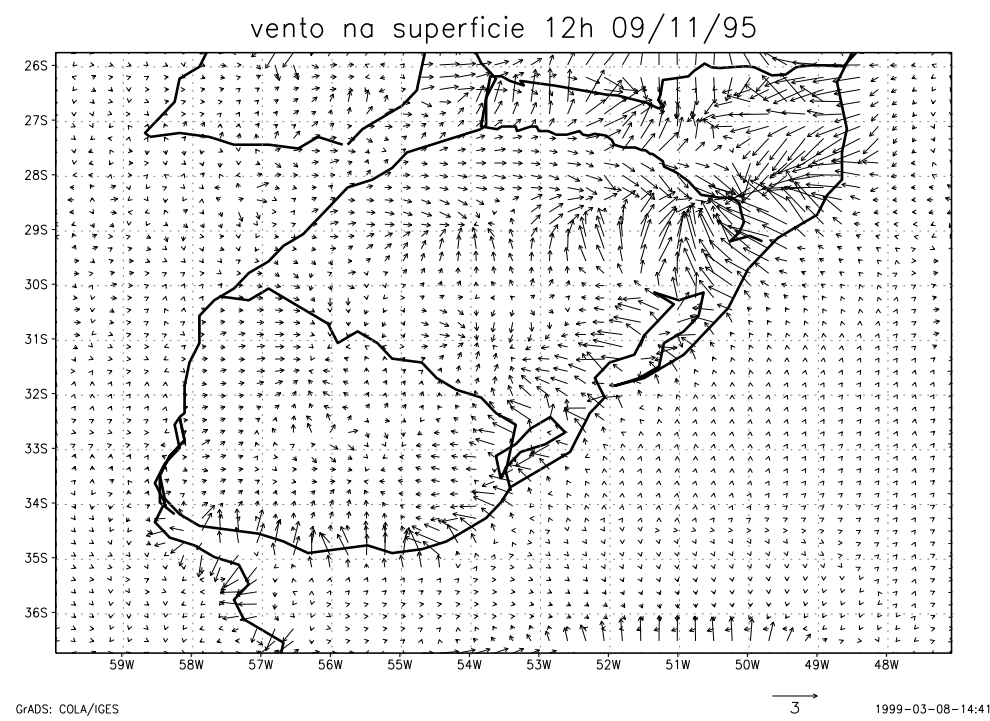

Figura 3: Campo de vento em superfície as 12 horas para o experimento 01

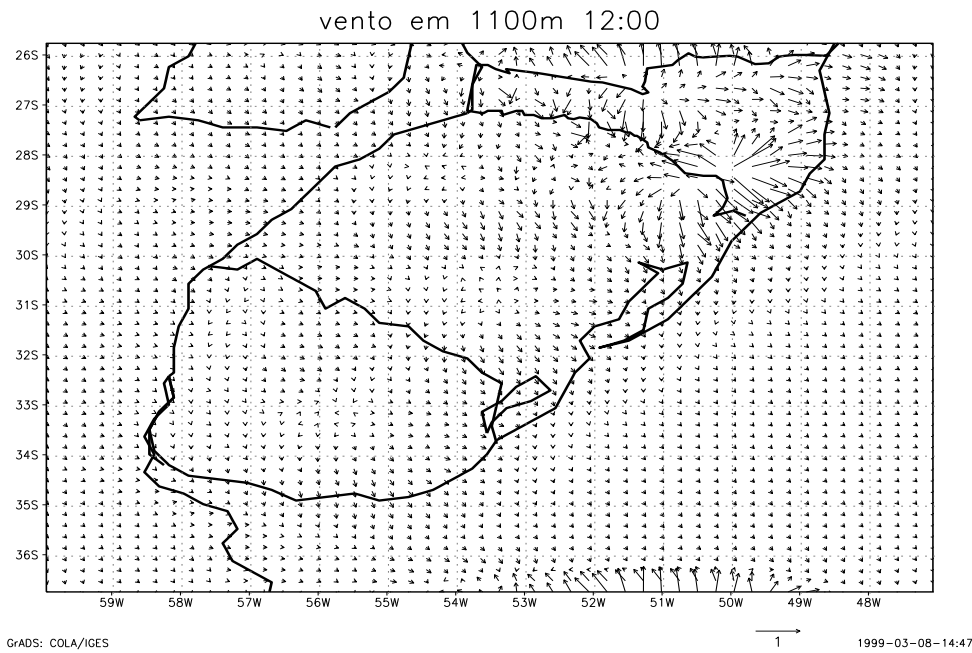

Figura 4: Campo de vento em 1100 metros as 12 horas para o experimento 01. 
Observa-se, da figura 3, a entrada da brisa em todo litoral simulado. Uma forte convergência, próximo a $29 \mathrm{~S}$ e $50 \mathrm{~W}$, é resultante da circulação causada pela brisa vale - montanha. Convergências mais fracas aparecem próximo a Candiota 31S e 54W e também no Uruguai 33S e 56W, devido a pequena variação na topografia. A brisa marítima e a forte convergência são circulações clássicas. As convergências menos intensas, em Candiota e na bacia do rio Uruguai são conhecidas como circulações não-clássicas.A figura 4 ilustra o campo de vento no horário das 12 horas na altura de 1100 metros. Pode-se observar, na linha litorânea, o fluxo de retorno da brisa marítima bem como uma acentuada divergência, em aproximadamente (50W, 28S) correspondente a forte convergência ocorrida na superfície.

A figura 5 a seguir ilustra o perfil de vento, em coordenadas Sigma-z (que seguem o terreno) para o corte vertical associado a figura 2.

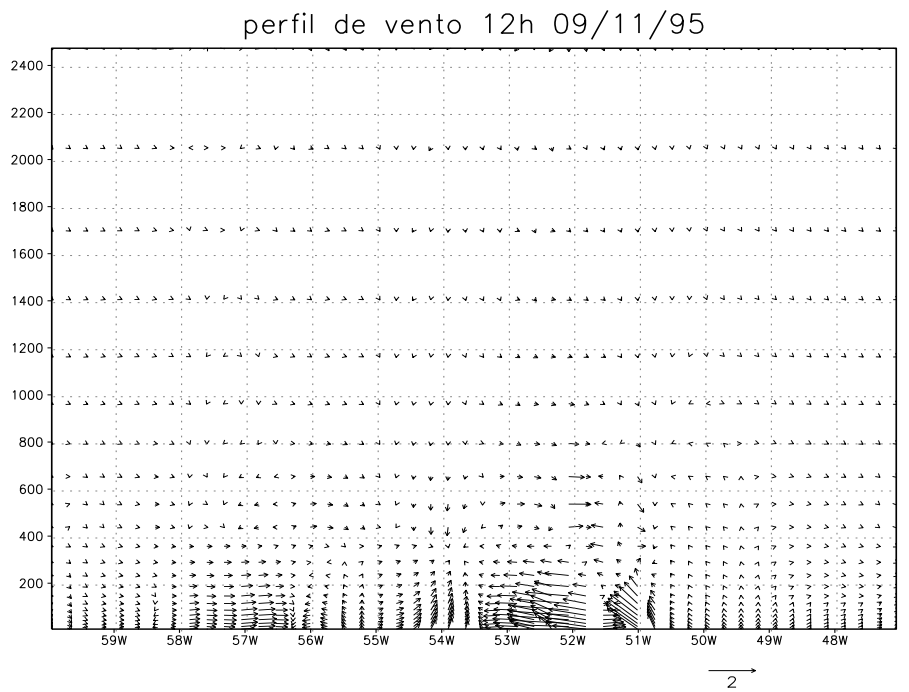

GrADS: COLA/IGES

$1999-03-08-14: 29$

Figura 5: Perfil do vento, as 12 horas, para o corte vertical na latitude de -31.3 (figura2).

Rev. Ciência e Natura - Dispersion Process: 93 - 111 ,2000. 
Observa-se, da figura acima, em $51.2 \mathrm{~W}$, uma convergência em superfície bem como uma divergência em aproximadamente 300 metros. Tais comportamentos são devido a penetração da brisa marítima e da brisa lacustre no continente (em superfície) e o escoamento de retorno em altitude.

As circulações geradas devido a pequena diferença de topografia e rugosidade do solo são também bem representadas pelo modelo. Verifica-se, entre $56 \mathrm{~W}$ e $53 \mathrm{~W}$, a circulação gerada pelo pequeno vale e a circulação gerada pelo rio Uruguai em aproximadamente $58.4 \mathrm{~W}$.

\section{Simulação com Inicialização Heterogênea .}

Neste experimento o RAMS é inicializado com as análises geradas pelo modelo global do CPTEC - INPE, (inicialização heterogênea),

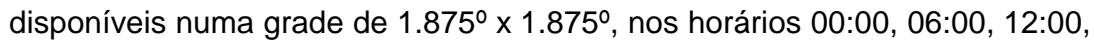
18:00 e 24:00 do dia 11 de Novembro de 1995. Nesta simulação foi utilizada a opção de vegetação variável. Entretanto, a umidade inicial do solo foi constante em todo domínio de integração do modelo. Os resultados mostrados nesta seção, que são comparados com dados coletados em campanhas experimentais, incluem os fluxos de calor e momentum e velocidade e direção do vento para o primeiro nível do modelo (10 metros) e para o ponto de grade correspondente a localização de Candiota Os dados observacionais foram coletados por sensores instalados a 10 metros em uma torre micrometeorológica. Detalhamento das campanhas experimentais encontra-se em Moraes (2000). As variáveis turbulentas foram amostradas com uma frequência de $10 \mathrm{~Hz}$ e mediadas a cada meia hora.

Nas figuras 6 e 7 encontra-se a variação temporal da direção e da velocidade do vento simuladas pelo RAMS e observadas na torre micrometeorológica. 
Observa-se dessas figuras que o RAMS simulou bem a direção do vento. Os dados medidos e os dados simulados pelo modelo permanecem de nordeste durante as 24 horas simuladas. Particularmente, 0 RAMS indica um vento proveniente, em todo perído, de um ângulo maior do que 45 graus, enquanto que a direção observada as 10, 15 e 19 horas é menor do que 45 graus. Importante observar, entretanto, é que a correlação entre estes dois parâmetros é maior do que $75 \%$. Em relação a velocidade do vento, figura 7 , notamos que o modelo subestima a velocidade do vento no final do período de simulação.

De maneira geral o modelo está suavizando os valores de velocidade em aproximadamente $2 \mathrm{~m} / \mathrm{s}$. Observe-se que, no período inicial, o modelo global do CPTEC/INPE fornece um vento de $7 \mathrm{~m} / \mathrm{s}$ e que após uma hora de integração o RAMS já indica um vento de 1,7 m/s. Pedro Dias (1999) sugere que uma melhor concordância entre velocidade simulada e observada pode ser obtida com um ajuste na subrotina onde o número de Richardson crítico é calculado.

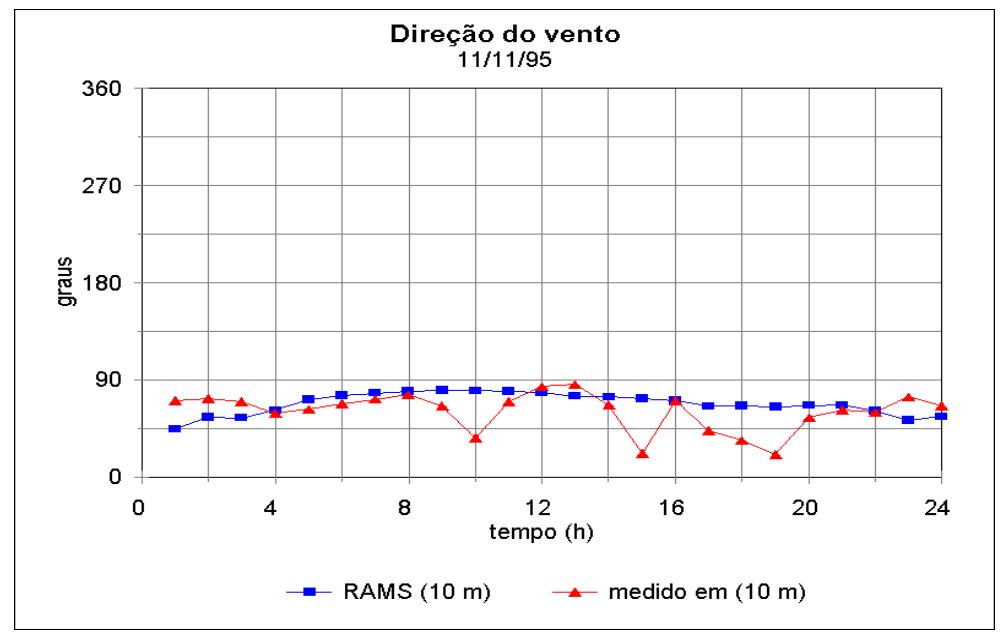

Figura 6 - Variação temporal da direção do vento em 10 metros.

Rev. Ciência e Natura - Dispersion Process: 93 - 111 ,2000. 


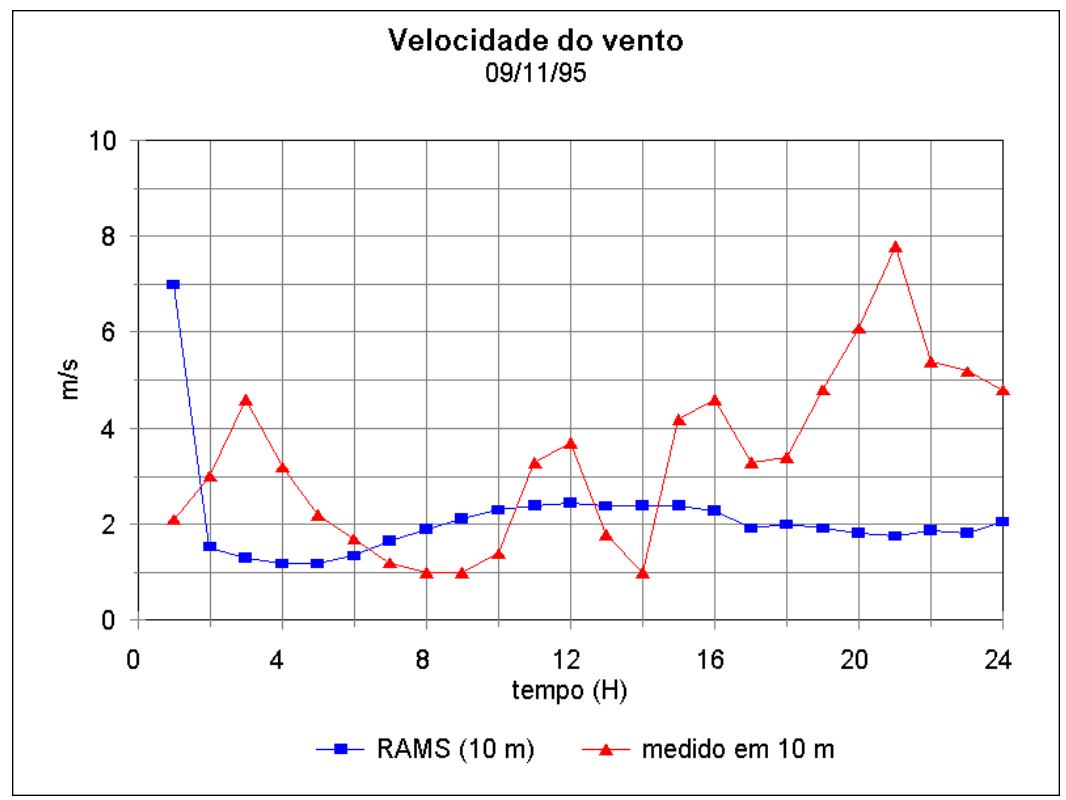

Figura 7- Velocidade do vento em 10 metros.

As figuras 8-10, a seguir, ilustram a variação temporal da velocidade de atrito, fluxo de calor latente e fluxo de calor sensível. Em todas as figuras os círculos representam dados observacionais e os triângulos valores simulados. As simulações correspondem ao ponto de grade de Candiota no primeiro nível do modelo. Em relação a velocidade de atrito nota-se que no período noturno existe uma correlação próximo a um entre observação e simulação. No período diurno o modelo subestima os valores observados. Certamente isto é uma decorrência da suavização do modelo para a velocidade, diminuindo assim a geração de turbulência por efeitos mecânicos. 


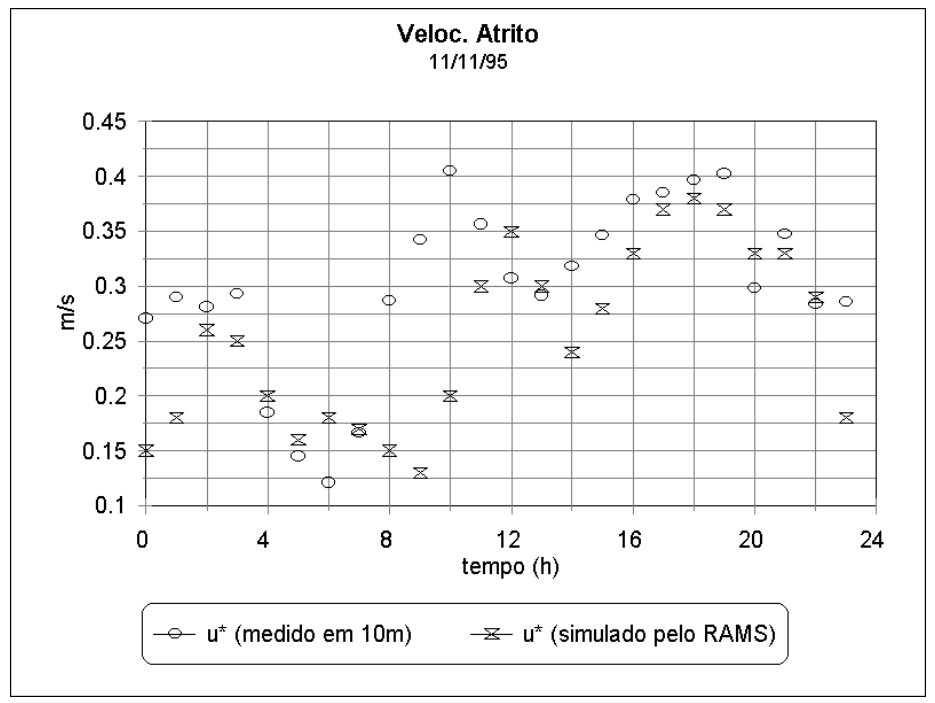

Figura 8- Velocidade de atrito em 10 metros.

Os fluxos superficiais de calor sensível $(H)$ e latente (LE) mostram uma boa concordância entre observação e simulação. Os máximos observados e simulados são da ordem de $400 \mathrm{~W} / \mathrm{m}^{2}$ e $200 \mathrm{~W} / \mathrm{m}^{2}$ para para $\mathrm{LE} \mathrm{e} \mathrm{H}$, respectivamente. Uma pequena diferença entre os dois conjuntos de dados, entre 12 e 13 horas, deve a uma pequena cobertura de nuvens que não está sendo resolvida pelo modelo. 


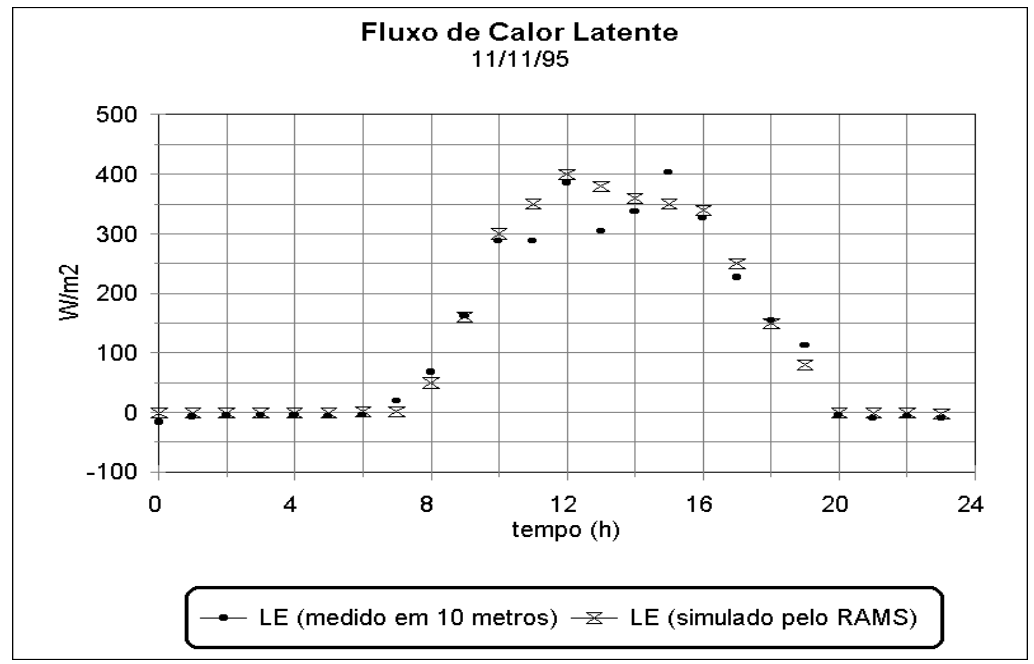

Figura 9: Fluxo de calor latente simulados pelo modelo RAMS e calculados através de dados experimentais medidos em 10 metros.

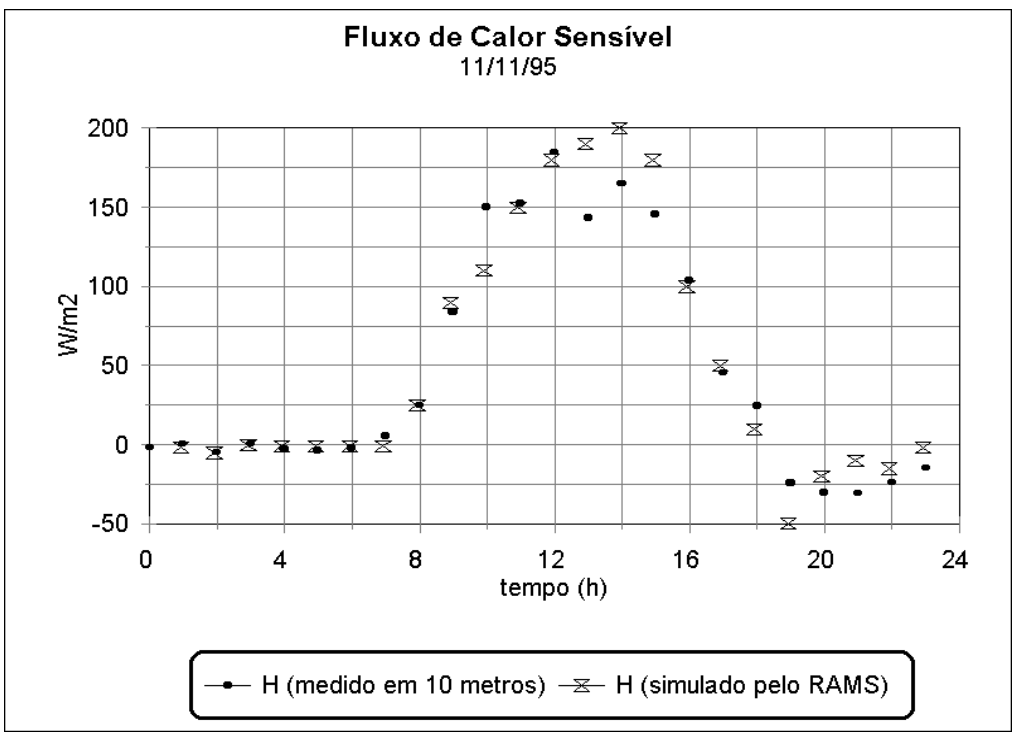

Figura 10: Fluxo de calor sensível simulados pelo modelo RAMS e calculados através de dados experimentais medidos em 10 metros.

Rev. Ciência e Natura - Dispersion Process: 93 - 111 ,2000. 


\section{CONCLUSÕES}

Neste trabalho simulou-se a circulação atmosférica com o RAMS no sul do Brasil, particularmente para uma grade centrada na latitude e longitude da Usina Termoelétrica de Candiota. Dois experimentos numéricos foram apresentados. O primeiro experimento, inicializado sem forçante externo, mostrou que o modelo é altamente sensível a mudanças topográficas. As circulações associadas a relevos, ainda que não acentuados, são descritas. Também, circulações originadas por gradientes térmicos podem ser distinguidas. As brisas marítima e lacustre são satisfatoriamente reproduzidas. Interessante observar que a circulação associada a lagoa causa um movimento subsidente sobre a mesma e ascendente na porção de terra localizada entre a lagoa e o oceano durante $o$ dia. A principal causa dessas circulações é o gradiente de capacidade térmica superficial.

Um segundo experimento, inicializado com dados advindos do modelo global do CPTEC/INPE teve seus resultados comparados com medidas obtidas em experimentos de campo. De modo geral os fluxos superficiais de momentum e calor são reproduzidos de maneira satisfatória. Em relação ao campo de vento mostrou-se que a direção predita pelo RAMS é aquela observada experimentalmente. Entretanto o módulo da velocidade é subestimada pelo modelo.

Agradecimentos: Trabalho parcialmente financiado pelas seguintes agências: Fundação de Amparo à Pesquisa do Estado de São Paulo (FAPESP), Conselho Nacional de Desenvolvimento Científico e Tecnológico (CNPq) e Fundação de Amparo à Pesquisa do Estado do Rio Grande do Sul (FAPERGS).

\section{REFERÊNCIAS BIBLIOGRÁFICAS}

Rev. Ciência e Natura - Dispersion Process: 93 - 111 ,2000. 
- Avissar R. and F. Chen (1993). Development and Analysis of Prognostic Equations for Mesoescale Kinetic Energy and Mesoescale (Subgrid Scale) Fluxes for Large-Scale Atmospheric Models, Journal of the Atmospheric Sciences, 50, 3751- 3774.

- Clark, T. L., (1977) A small-scale dynamic model using a terrain-following coordinate transformation. J. Comp. Phys., 24, pp. 186-215.

- Chen, C., and W. R. Cotton (1983) A One-Dimensional Simulation of the Stratocumulus-Capped Mixed Layer. Boundaty Layer Meteorology, 25, pp. 289-321.

- Cotton, W. R., Tripoli, G. J. (1978). Cumulus convection in Shear Flow Three-Dimensional Numerical Experiments. J. Atmos. Sci., 35, 15031521.

- McCumber, M. C., and R. A. Pielke (1981). Simulation of the Effects of the Surfece Fluxes of Heat and Moisture in a Mesoescale Numerical Model. Part I: Soil Layer. J. Geophys+. Res., 86, pp. 9929-9938.

- Moraes, O L. L., Oliveira, A P., Neto, E. C. e Degrazia, G.A., Air Pollution: The Candiota Field Program, 125pp (1996)

- Moraes, O. L. L. (2000), Turbulence Characteristics in the Surface Boundary Layer over the South America Pampa, aceito para publicação na Boundary Layer Meteorology.

- Pielke, R. A., Cotton, W. R., Walko L. R., Tremback C. J., Lyons W. A., Grasso L. D., Nicholls M. E., Moran M. D., Wesley D. A., Lee T. J., Copeland J. H., (1992). A Comprehensive Meteorological Modeling System - RAMS. Meteorological Atmos. Phys. 69-90.

- Silva Dias, P., comunicação pessoal (1999). 
- Smagorinsky, J. (1963). General Circulation Experiments with the Primitive Equations. 1: The Basic Experiment. Mon. Weather Rev., 91, pp. 99-164

- Stull, R.B., 1988: An Introduction to Boundary Layer Meteorology, Kluwer Academic Publishers, Dordrecht, Holanda.

- Tremback, C. J., J. Powell, W. R. Cotton and R. Pielke (1987). The Forward-in-Time Upstream Scheme: Extension to Higher Orders. Mon. Wea. Rev., 115, pp. 540-555

- Tremback, C. J., and R. Kessler (1985). A Surfece Temperature and Moisture Parametrization for use in Mesoescale Nemerical Models. Preprints, 7 th. Conference on Numerical Weather Prediction, Montral, Canada, MAS, 17-20 June 1985.

- Tremback, C. J., (1990). Numerical Simulation of a Mesoescale Convective Complex: Model Development and Numerical Results. Department of Atmospheric Science. Colorado State University.

- Tripoli, G. J. and W. R. Cotton (1980). A Numerical Investigation of Several Factors Leading to the Observed Variable Intensity of Deep Convection over South Florida. J. Appl. Meteor., 19, 1037-1063.

- Tripoli, G. J. and W. R. Cotton (1982). The Colorado State University Tree-Dimensional Cloud / Mesoescale Model. Parte I: General Theorical Framework and Sensitivity Experiments. J. Recherches Atmos., 16, pp. 186-219. 
112 Rev. Ciência e Natura - Dispersion Process: 93 - 2000. 